GSA Data Repository 2017240

Fire biases the production of charred flowers: Implications for the Cretaceous fossil record

Victoria A. Hudspith ${ }^{1}$, and Claire M. Belcher ${ }^{1}$

${ }^{1}$ wildFIRE lab, Hatherly Laboratories, Department of Geography, University of Exeter, Exeter, EX4 4PS, Devon, UK

\title{
CONTENT INCLUDES
}

Supplementary Tables DR1, DR2 
Table DR1 Summary of four angiosperm species where entire inflorescences, not individual flowers were counted pre- and post-burn. Details include inflorescence preservation potential as charcoal, and calorimetry test information.

\begin{tabular}{|c|c|c|c|c|c|c|c|c|c|c|c|c|c|}
\hline $\begin{array}{l}\text { Family } \\
\text { name }\end{array}$ & $\begin{array}{l}\text { Taxa tested } \\
\text { (inflorescence } \\
\text { type in } \\
\text { brackets) }\end{array}$ & $\begin{array}{l}\text { Number } \\
\text { of } \\
\text { infloresce } \\
\text { nces at } \\
\text { start of } \\
\text { test }\end{array}$ & $\begin{array}{l}\text { Number of } \\
\text { inflorescenc } \\
\text { es at end of } \\
\text { test }\end{array}$ & $\begin{array}{l}\text { charred } \\
\text { inflores } \\
\text { cences } \\
(\%)\end{array}$ & $\begin{array}{l}\text { Start } \\
\text { mass } \\
\text { (g) }\end{array}$ & $\begin{array}{l}\text { End } \\
\text { mass } \\
\text { (g) }\end{array}$ & $\begin{array}{l}\text { Mass } \\
\text { lost } \\
(\%)\end{array}$ & $\begin{array}{l}\text { Time to } \\
\text { Ignition } \\
\text { (s) }\end{array}$ & $\begin{array}{l}\text { End of } \\
\text { Test (s) }\end{array}$ & $\begin{array}{l}\text { Flaming } \\
\text { duration } \\
\text { (s) }\end{array}$ & $\begin{array}{l}\text { Peak } \\
\text { heat } \\
\text { release } \\
\text { rate } \\
\left(\mathrm{kW} / \mathrm{m}^{2}\right)\end{array}$ & $\begin{array}{l}\text { Time } \\
\text { to } \\
\text { pHRR } \\
\text { (s) }\end{array}$ & $\begin{array}{l}\text { Time } \\
\text { to } \\
\text { peak } \\
\text { MLR } \\
\text { (s) }\end{array}$ \\
\hline \multirow{5}{*}{ 离 } & \multirow{3}{*}{$\begin{array}{l}\text { Spathiphyllum } \\
\text { wallisii } \\
\text { (spadix) }\end{array}$} & 7 & 7 & 100 & 3.71 & 0.49 & 87 & 3 & 69 & 66 & 285.75 & 5 & 5 \\
\hline & & 5 & 4 & 80 & 3.17 & 0.49 & 85 & 4 & 52 & 48 & 201.31 & 5 & 6 \\
\hline & & 8 & 5 & 63 & 4.26 & 0.72 & 83 & 7 & 69 & 62 & 196.08 & 10 & 9 \\
\hline & \multirow{2}{*}{$\begin{array}{l}\text { Calla palustris } \\
\text { (spadix) }\end{array}$} & 2 & 2 & 100 & 3.59 & 0.63 & 82 & 6 & 17 & 11 & 369.95 & 7 & 8 \\
\hline & & 4 & 4 & 100 & 3.13 & 0.45 & 86 & 5 & 24 & 19 & 254.34 & 6 & 7 \\
\hline \multirow{3}{*}{ 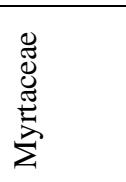 } & \multirow{3}{*}{$\begin{array}{l}\text { Callistemon } \\
\text { salignus } \\
\text { (spike) }\end{array}$} & 6 & 2 & 33 & 16.08 & 2.34 & 85 & 13 & 143 & 130 & 624.53 & 13 & 15 \\
\hline & & 7 & 2 & 29 & 16.12 & 2.36 & 85 & 20 & 140 & 120 & 663.79 & 21 & 22 \\
\hline & & 6 & 2 & 33 & 12.86 & 1.52 & 88 & 10 & 140 & 130 & 623.6 & 13 & 13 \\
\hline \multirow{3}{*}{ 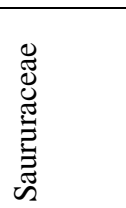 } & \multirow{3}{*}{$\begin{array}{l}\text { Anemopsis } \\
\text { californica } \\
\text { (spike) }\end{array}$} & 6 & 6 & 100 & 7.88 & 1.46 & 81 & 15 & 32 & 17 & 360.39 & 17 & 17 \\
\hline & & 9 & 9 & 100 & 8.95 & 1.82 & 80 & 13 & 34 & 21 & 369.02 & 15 & 15 \\
\hline & & 9 & 8 & 89 & 8.23 & 1.62 & 80 & 8 & 32 & 24 & 399.38 & 13 & 12 \\
\hline
\end{tabular}


Table DR2 Summary table of the remaining nine angiosperm species tested, including details on each inflorescence and individual charred flower preservation as well as calorimetry test information.

\begin{tabular}{|c|c|c|c|c|c|c|c|c|c|c|c|c|c|}
\hline $\begin{array}{l}\text { Family } \\
\text { name }\end{array}$ & $\begin{array}{l}\text { Taxa tested } \\
\text { (inflorescence } \\
\text { type in } \\
\text { brackets) }\end{array}$ & $\begin{array}{l}\text { Number } \\
\text { of } \\
\text { flowers } \\
\text { at start of } \\
\text { test }\end{array}$ & $\begin{array}{l}\text { Number of } \\
\text { flowers at } \\
\text { end of test }\end{array}$ & $\begin{array}{l}\text { charred } \\
\text { flowers } \\
(\%)\end{array}$ & $\begin{array}{l}\text { Start } \\
\text { mass } \\
\text { (g) }\end{array}$ & $\begin{array}{l}\text { End } \\
\text { mass } \\
\text { (g) }\end{array}$ & $\begin{array}{l}\text { Mass } \\
\text { lost } \\
\text { (\%) }\end{array}$ & $\begin{array}{l}\text { Time to } \\
\text { Ignition } \\
\text { (s) }\end{array}$ & $\begin{array}{l}\text { End of } \\
\text { Test (s) }\end{array}$ & $\begin{array}{l}\text { Flaming } \\
\text { duration } \\
\text { (s) }\end{array}$ & $\begin{array}{l}\text { Peak } \\
\text { heat } \\
\text { release } \\
\text { rate } \\
\left(\mathbf{k W} / \mathbf{m}^{2}\right)\end{array}$ & $\begin{array}{l}\text { Time } \\
\text { to } \\
\text { pHRR } \\
\text { (s) }\end{array}$ & $\begin{array}{l}\text { Time } \\
\text { to } \\
\text { peak } \\
\text { MLR } \\
\text { (s) }\end{array}$ \\
\hline \multirow[b]{2}{*}{ 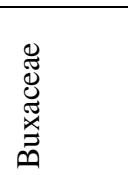 } & \multirow{2}{*}{$\begin{array}{l}\text { Pachysandra } \\
\text { terminalis } \\
\text { (spike) }\end{array}$} & 72 & 8 & 11 & 9.08 & 0.93 & 90 & 15 & 44 & 29 & 571.82 & 19 & 17 \\
\hline & & 35 & 23 & 66 & 9.42 & 1.06 & 89 & 13 & 37 & 24 & 648.66 & 19 & 16 \\
\hline \multirow{6}{*}{ 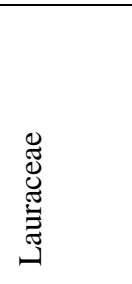 } & \multirow{3}{*}{$\begin{array}{l}\begin{array}{l}\text { Laurus nobilis } \\
\text { (cymose/ }\end{array} \\
\text { umbelliform) }\end{array}$} & 460 & 65 & 14 & 9.78 & 0.8 & 92 & 8 & 68 & 60 & 662.2 & 13 & 10 \\
\hline & & 300 & 218 & 73 & 10.3 & 1.11 & 89 & 7 & 29 & 22 & 828.48 & 9 & 9 \\
\hline & & 455 & 251 & 55 & 9.3 & 0.76 & 92 & 6 & 10 & 4 & 779.57 & 10 & 8 \\
\hline & \multirow{3}{*}{$\begin{array}{l}\text { Cinnamomum } \\
\text { camphora } \\
\text { (paniculate) }\end{array}$} & 36 & 22 & 61 & 5.41 & 0.58 & 89 & 4 & 11 & 7 & 603.86 & 6 & 7 \\
\hline & & 113 & 5 & 4 & 5.68 & 0.61 & 89 & 4 & 7 & 3 & 649.05 & 7 & 6 \\
\hline & & 137 & 10 & 7 & 6.02 & 0.66 & 89 & 4 & 14 & 10 & 621.98 & 8 & 6 \\
\hline \multirow{4}{*}{ 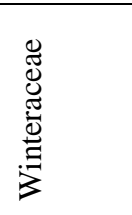 } & \multirow{4}{*}{$\begin{array}{l}\text { Drimys winteri } \\
\text { (umbel) }\end{array}$} & 18 & 8 & 44 & 5.9 & 0.6 & 90 & 16 & 24 & 8 & 522.79 & 19 & 18 \\
\hline & & 20 & 6 & 30 & 7.76 & 0.83 & 89 & 9 & 37 & 28 & 617.55 & 14 & 11 \\
\hline & & 18 & 7 & 39 & 9.27 & 0.87 & 91 & 15 & 32 & 17 & 735.99 & 16 & 17 \\
\hline & & 15 & 4 & 27 & 13.83 & 1.83 & 87 & 20 & 62 & 42 & 671.57 & 24 & 23 \\
\hline \multirow[b]{2}{*}{ 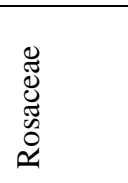 } & \multirow{2}{*}{$\begin{array}{l}\text { Sorbaria } \\
\text { kirilowii } \\
\text { (paniculate) }\end{array}$} & 584 & 520 & 89 & 4.36 & 0.72 & 83 & 8 & 9 & 1 & 395.98 & 9 & 9 \\
\hline & & 773 & 639 & 83 & 5.29 & 0.79 & 85 & 11 & 20 & 9 & 325.93 & 13 & 14 \\
\hline \multirow[t]{2}{*}{ 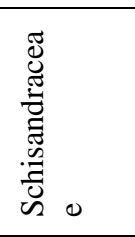 } & $\begin{array}{l}\text { Illicium cf. } \\
\text { henryi } \\
\text { (None: solitary } \\
\text { flowers) }\end{array}$ & 2 & 2 & 100 & 9.84 & 0.88 & 91 & 3 & 33 & 30 & 770.42 & 9 & 10 \\
\hline & Schisandra & 15 & 10 & 67 & 5.04 & 0.55 & 89 & 22 & 33 & 11 & 278.13 & 23 & $23^{3}$ \\
\hline
\end{tabular}




\begin{tabular}{|c|c|c|c|c|c|c|c|c|c|c|c|c|c|}
\hline & $\begin{array}{l}\text { rubriflora } \\
\text { (None: solitary } \\
\text { flowers) }\end{array}$ & 29 & 25 & 86 & 6.37 & 0.94 & 85 & 5 & 21 & 16 & 395.67 & 8 & 7 \\
\hline \multirow{2}{*}{ 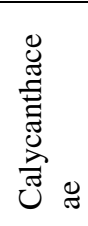 } & \multirow{2}{*}{$\begin{array}{l}\text { Calycanthus } \\
\text { occidentalis } \\
\text { (None: solitary } \\
\text { flowers) }\end{array}$} & 2 & 2 & 100 & 5.09 & 1.39 & 73 & 6 & 8 & 2 & 472.66 & 8 & 8 \\
\hline & & 3 & 3 & 100 & 5.8 & 1.03 & 82 & 6 & 23 & 17 & 510.68 & 8 & 9 \\
\hline \multirow{3}{*}{ 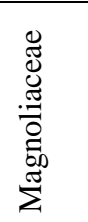 } & \multirow{3}{*}{$\begin{array}{l}\text { Liriodendron } \\
\text { tulipifera } \\
\text { (None: solitary } \\
\text { flowers) }\end{array}$} & 2 & 2 & 100 & 7.75 & 1.16 & 85 & 9 & 93 & 84 & 339.4 & 10 & 11 \\
\hline & & 3 & 1 & 33 & 12.14 & 3.18 & 74 & 4 & 102 & 98 & 397.87 & 6 & 7 \\
\hline & & 4 & 2 & 50 & 11.6 & 1.85 & 84 & 6 & 114 & 108 & 437.99 & 9 & 9 \\
\hline
\end{tabular}

